\title{
Alterstice
}

Revue internationale de la recherche interculturelle

International Journal of Intercultural Research

Revista International de la Investigacion Intercultural

\section{Un lectorat en hausse constante}

\section{Yvan Leanza}

Volume 4, numéro 2, 2014

URI : https://id.erudit.org/iderudit/1077420ar

DOI : https://doi.org/10.7202/1077420ar

Aller au sommaire du numéro

Éditeur(s)

Alterstice

ISSN

1923-919X (numérique)

Découvrir la revue

Citer ce document

Leanza, Y. (2014). Un lectorat en hausse constante. Alterstice, 4(2), 1-2.

https://doi.org/10.7202/1077420ar d'utilisation que vous pouvez consulter en ligne.

https://apropos.erudit.org/fr/usagers/politique-dutilisation/ 
ÉDITORIAL

\title{
Un lectorat en hausse constante
}

\author{
Yvan Leanza $^{1}$
}

\begin{abstract}
Sans donner aux chiffres de pouvoirs magiques absolus, il est nécessaire, aujourd'hui, pour un comité d'édition, de connaître quelques faits sur le lectorat de sa revue. Nous avons effectué l'exercice récemment et je profite de cet éditorial pour en partager les points les plus saillants. Les analyses sont faites à partir des données du site de la revue recueillies avec Webalizer 2.01. Elles ont été possibles grâce à l'assistance éclairée de François René de Cotret, étudiant au doctorat en psychologie à l'Université Laval (Québec). Merci à lui! En bref, la revue est lue, voire très lue!
\end{abstract}

Alterstice a vu son nombre de lecteurs augmenter graduellement depuis son lancement. Alors que le site Internet attirait quelque 1000 visiteurs mensuels en décembre 2011, date de la mise en ligne du premier numéro, ils ont été plus de 8000 à s'y rendre en décembre 2014.

La parution d'un nouveau numéro de la revue s'accompagne d'une recrudescence immédiate du lectorat. La montée en flèche du nombre de visites depuis la parution du dernier numéro, en septembre 2014, est particulièrement remarquable : en août 2014, environ 36000 pages ont été visitées, alors que ce sont près de 62000 qui l'ont été le mois suivant, et, depuis, la moyenne est d'environ 50000 pages par mois. Avec une centaine de publications, dont 57 articles évalués par les pairs et 8 numéros complets, le nombre total de publications disponibles semble être une base suffisante pour attirer régulièrement de nouveaux lecteurs. En effet, bien que les articles les plus récents soient parmi les plus consultés par les visiteurs du site, les anciens numéros demeurent populaires. Chaque article évalué par les pairs est téléchargé en moyenne 1,4 fois par jour, 4,5 fois par jour en tenant compte du téléchargement de chaque numéro intégral.

Non seulement les publications d'Alterstice attirent de plus en plus de lecteurs, mais ceux-ci proviennent de pays de plus en plus variés. De 31 pays en décembre 2011, le trafic d'Alterstice était représenté par 59 pays en décembre 2014.

Ces articles sont lus, certes, mais sont-ils cités ou employés? Google Scholar permet une première analyse : selon cette source, le tiers des articles d'Alterstice sont cités. Par ailleurs, l'accès libre et immédiat suscite un phénomène intéressant : plusieurs sites académiques rendent disponibles les articles d'Alterstice en format PDF ou renvoient directement au lien de téléchargement sur Alterstice. Une recherche effectuée sur le moteur de recherche Google permet de constater que ce phénomène touche la majorité des articles publiés, à l'exception du numéro le plus récent.

Afin de bonifier cette analyse, une invitation à participer à un sondage a été envoyée aux 367 " abonnés " d'Alterstice le 5 mai 2014 : 76 personnes, provenant de 14 pays, ont répondu. Les deux tiers (65 \%) des répondants affirment avoir utilisé certains articles révisés par les pairs dans le cadre de leur enseignement au moins une fois au cours des deux dernières années (figure 1). Les commentaires des répondants montrent que le tiers restant n'enseigne pas et ne fait pas non plus de recherche, mais est dans la pratique. Ces personnes lisent la revue pour se tenir à jour. En outre, $61 \%$ des répondants disent avoir cité au moins un article d'Alterstice dans leurs propres 
articles soumis à des revues savantes au cours des deux dernières années. Enfin, la majorité des répondants (56 \%) auraient utilisé les articles d'Alterstice à d'autres fins, par exemple en tant que références dans un ouvrage ou une activité de transfert de connaissance.

Figure 1 : Fréquence d'utilisation des articles revus par les pairs par les abonnés d'Alterstice

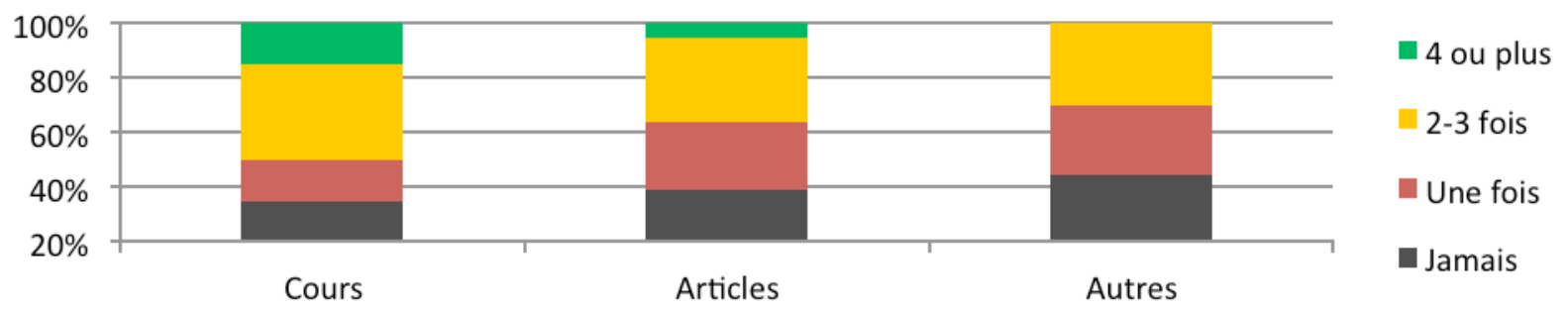

En résumé, Alterstice, avec des moyens modestes, réussit à atteindre un public international. Le format de libre accès immédiat rend la revue disponible non seulement pour des chercheurs, mais aussi pour des enseignants et des praticiens de régions du monde moins favorisées, ce qui est conforme au principe énoncé lors de sa création.

Avant les chiffres, il y a des contenus et des humains... La revue ne pourrait exister sans les nombreuses personnes, expertes de leur champ, qui donnent de leur temps pour évaluer les articles soumis. Qu'elles en soient ici chaleureusement remerciées! Au cours des deux dernières années, ont ainsi contribué grandement à la qualité des articles publiés :

Azzam Amin; Claire Autan-Dorier; Anne-Marie Bellemare; Alexandra Boilard; Isabelle Boisclair; Olivier-Serge Candau; Nicole Carignan; Nilima Changkakoti; Geneviève Cloutier; Beate Collet; Viviane Cretton; Patrick Dramé; Khadiyatoulah Fall; Jacques Forget; Sarah Fraser; Séverine Garnier; François Gauthier; Betty Goguikian; Aline Gohard; Zohra Guerraoui; Ghayda Hassan; Félicia Heidenreich Dutray; Audrey Heine; André Jacob; Estibaliz Jimenez; Yasser Khazaal; Mondher Kilani; Julia Krane; Jean Lafontant; Myrna Lashley; Josianne Le Gall; Annabel Levesque; Sue-Ann MacDonald; Alexis Martig; Lucienne Martins Borges; Raymond Massé; Toby Measham; Abdelwahed Mekki-Berrada; Claire Mestre; Thomas Michaud Labonté; René Mokounkolo; Lise Montminy; Vrinda Narain; Garine Papazian; Michèle Poitras; Maryse Potvin; Lilyane Rachédi; Jean Ramdé; Élodie Razy; Rhéa Rocque; Jules Rocque; Ilario Rossi; Cécile Rousseau; Andrew Ryder; Colette Sabatier; Sara Skandrani; Pascal Tisserant; Marcel Trudel; Daniel Turcotte; Michèle Vatz Laaroussi; Bilkis Vissandjee; Bob White; Margareth Zanchetta.

On peut souhaiter que le présent numéro ait autant de succès que les précédents. Le thème est d'importance : santé mentale et sociétés plurielles. Betty Goguikian (Université de Genève, Suisse) et Ilario Rossi (Université de Lausanne, Suisse) ont réuni autour de ce thème des experts de disciplines multiples, ce qui permet une lecture interculturelle originale et critique.

Bonne lecture!

\section{Rattachement de l'auteur}

${ }^{1}$ Université Laval, Québec (Québec), Canada

\section{Correspondance}

alterstice@gmail.com

\section{Pour citer cet article}

Leanza, Y. (2014). Un lectorat en hausse constante [Éditorial]. Alterstice, 4(2), 1-2. 